\title{
White Thrombosis on Optical Coherence Tomography after Rotational Atherectomy of Severely Coronary Calcified Lesions
}

\section{Cheng-fu CAO}

Peking University People's Hospital

\section{Yu-liang MA}

Peking University People's Hospital

Qi LI

Peking University People's Hospital

Jian LIU

Peking University People's Hospital

\section{Hong ZHAO}

Peking University People's Hospital

\section{Ming-yu LU}

Peking University People's Hospital

weimin wang ( $\nabla$ weiminwang@vip.sina.com )

Peking University People's Hospital https://orcid.org/0000-0003-4953-0777

\section{Research article}

Keywords: coronary calcification, rotational atherectomy, white thrombosis, optical coherence tomography

Posted Date: September 16th, 2020

DOI: https://doi.org/10.21203/rs.3.rs-19367/v2

License: (a) (1) This work is licensed under a Creative Commons Attribution 4.0 International License. Read Full License 


\section{Abstract}

Background: Rotational atherectomy (RA) has improved percutaneous treatment of severe coronary calcified lesions, but the "no-reflow" phenomenon remains a serious complication. Platelet activation by RA may contribute to the no-reflow, we use optical coherence tomography (OCT) to test the effect of RA on white thrombosis that could confirm platelet activation indirectly.

Methods: We analyzed 53 consecutive patients with coronary angiographic severe calcified lesions. All the patients were examined by OCT. Twenty patients received RA and OCT imaging performed before and after RA and after stent implantation. The remaining patients were as a control group and OCT imaging performed before balloon dilatation and after stent implantation.

Results: The patients were older and higher incidence of Diabetes mellitus in the RA group. In the RA group, the mean burr size was $1.48 \pm 0.14 \mathrm{~mm}$. The mean rotation speed was $152,300 \pm 4,200 \mathrm{rpm}$. The mean number of rotations per patient was $5.3 \pm 2.1$ times and the mean ablation time per RA was $11.3 \pm 3.2$ seconds. In the control group, there was no thrombogenesis during the procedure, but in the RA group, all the target vessels had only white thrombosis on OCT after RA. The average number of white thrombus per lesion after RA was $7.23 \pm 4.4$, and the average length of every white thrombus was $0.51 \pm 0.33 \mathrm{~mm}$. In Pearson Correlation Analysis, Thrombotic load was related with burr size $(r=0.575$, $P=0.040)$ and rotation number $(r=0.599, P=0.031)$.

Conclusions: White thrombosis during RA is proved by OCT in vivo. Treating calcified lesions with RA may enhance thrombogenesis. These data suggest using a proper therapy to avoid no-reflow during RA.

\section{Background}

Coronary calcification plaque is common in patients undergoing percutaneous coronary interventions (PCI). The incidence ranges between $17 \%$ and $35 \%$ [1]. Coronary calcification affects the surgery success rate and long-term clinical outcome [2, 3]. Calcified lesions can lead to stent implantation failure or incomplete stent expansion, thus affecting the long-term efficacy of stents. It also increases the risk of perforation and coronary dissection during operation $[4,5]$. Rotational atherectomy (RA) plays a key role in the therapy of severely calcified lesions $[6,7]$. It can reduce the incidence of complications and improve the operation success rate. European expert consensus on rotational atherectomy recommend that when heavily calcified lesions that cannot be adequately dilated by a balloon before stenting, RA is a reasonable choice to sufficient pretreatment of calcified lesions [7].

Platelet activation is an important determinant of acute outcomes of $\mathrm{PCl}$. Some studies had confirmed that RA could cause platelet aggregation in vitro models [8,9], which lead to slow flow or no reflow during RA [10].

Optical coherence tomography (OCT) is a high resolution (10 $\mu \mathrm{m}$ axial), light-based imaging modality, it can accurately display the microstructure characteristics of atherosclerotic plaque, identify vulnerable 
plaque, thrombus, calcification and dissection, which has been demonstrated to show excellent correlation with histology [11]. To our knowledge, there has been no imaging study in vivo to assess the effect of RA on thrombogenesis by OCT. The purpose of our research was to use OCT to examine the effect of RA on thrombogenesis which could confirm platelet activation indirectly in vivo.

\section{Methods}

\section{Study population}

This is a single-center observational study, including 53 consecutive patients who underwent OCT imaging due to severe coronary calcification on angiography from July 2019 to December 2019. The definition of severe calcification on angiography was defined as clear calcified shadows that can be seen in both beating and resting hearts before contrast injection. If the OCT image shows a maximum calcium angle $>180^{\circ}$, maximum calcium thickness $>0.5 \mathrm{~mm}$ and maximum calcium length $>5 \mathrm{~mm}$, the patients will receive RA therapy before stent placement [12]. If the OCT catheter did not pass the lesion, OCT was performed immediately after dilation with a small balloon. Finally, 20 patients met the above criterion and were included in the RA group, and they received RA therapy before stent placement. The other 33 patients were included in the control group, and they received routine interventional therapy. The demographic and clinical characteristics of all patients were derived from the electronic record system.

This study was approved by the ethics at Peking University People's Hospital. All patients provided written informed consent before participation.

\section{RA details}

Before procedural, all patients received an oral loading dose of $300 \mathrm{mg}$ aspirin and $600 \mathrm{mg}$ clopidogrel. During procedural, all patients received unfractionated heparin at a dose of $70-100 \mathrm{U} / \mathrm{kg}$ or bivalirudin to maintain an activated clotting time (ACT) $>300$ seconds. The choice of vascular access, burr size was left at the operators' discretion. RA was performed by using the Rotablator (Boston Scientific Scimed, Inc., Maple Grove, MN, USA). The burr size was selected to reach a burr/vessel ratio of 0.5-0.6. RA speed ranged between 140,000 and 160,000 rotation per minute. Each ablation time was 10-15 seconds. During RA, a continuous intracoronary infusion of a cocktail with unfractionated heparin and nitroglycerin was employed. The success of RA was defined as the complete expansion of the balloon of the target lesion after RA.

\section{OCT Image Acquisition and Analysis}

OCT image acquisition was performed before RA, after RA and after stent placement in the RA group, and it was performed before balloon dilatation and after stent implantation in the control group with C7-XR or ILUIMEN OPTIS OCT Intravascular Imagining System (Abbott, USA). All OCT data were analyzed using Abbott Vascular Offline Review Workstation by two independent interventional cardiologists who were blinded to the clinical and angiographic information. 
The minimum lesion lumen area (MSA) was defined as the smallest cross-sectional area (CSA) in the lesion segment. Calcium lesion was evaluated using three parameters: maximum angle, maximum thickness, and length. When calcium was extremely thick, and its border was not clear due to attenuation, the maximum visible thickness was measured [12].

Thrombus was one consequence of platelet aggregation, so we confirmed platelet aggregation by thrombogenesis indirectly. White thrombus was defined as a mass attached to the luminal surface or floating within the lumen, which is less backscattering, homogeneous, and has low attenuation [13]. The thrombotic load was evaluated by the number of white thrombus on OCT.

\section{Statistical Analysis}

Descriptive statistics are presented as mean $\pm S D$ and compared with the $t$-test. Categorical data are expressed as frequency and percentage and was compared with chi-square or Fisher exact test where appropriate. Pearson Correlation Analysis was used to analyze the correlation between continuous variables.

\section{Results}

\section{Patient Characteristics}

We finely included 53 consecutive cases. baseline clinical characteristics were summarized in Table 1. The patients were older and had a higher incidence of Diabetes mellitus in the RA group. In the RA group, the average age of the patients was $70.3 \pm 9.5$ years. Most patients were male $(75.0 \%)$, and clinical manifestations were acute coronary syndrome (ACS).

Table 1. Baseline patients Characteristics 


\begin{tabular}{lccc}
\hline & $\begin{array}{c}\text { RA group } \\
(\mathrm{n}=20)\end{array}$ & $\begin{array}{c}\text { Control group } \\
(\mathrm{n}=33)\end{array}$ & $P$ value \\
\hline Age (years) & $70.3 \pm 9.5$ & $65.4 \pm 8.7$ & 0.004 \\
Male gender, n (\%) & $14(70.0 \%)$ & $22(66.7 \%)$ & 0.625 \\
Body mass index, (Kg/m²) & $25.7 \pm 4.2$ & $25.1 \pm 3.8$ & 0.912 \\
Hypertension, n (\%) & $12(60.0 \%)$ & $21(63.6 \%)$ & 0.853 \\
Hyperlipidemia, n (\%) & $7(35.0 \%)$ & $10(30.3 \%)$ & 0.621 \\
Diabetes mellitus, n (\%) & $11(55.0 \%)$ & $12(36.3 \%)$ & 0.024 \\
Smoking, n (\%) & $7(35.0 \%)$ & $13(39.4 \%)$ & 0.536 \\
Prior PCI, n (\%) & $3(15.0 \%)$ & $2(6.1 \%)$ & 0.134 \\
Prior CABG, n (\%) & $3(15.0 \%)$ & $2(6.1 \%)$ & 0.134 \\
LDL-c (mmol/L) & $2.2 \pm 1.0$ & $2.1 \pm 1.0$ & 0.745 \\
eGFR (ml/Kg.1.73m²) & $86.3 \pm 12.9$ & $89.2 \pm 11.2$ & 0.712 \\
Type of CAD & & & \\
SA, n (\%) & $5(25.0 \%)$ & $8(24.2 \%)$ & 0.836 \\
UA, n (\%) & $10(50.0 \%)$ & $16(48.5 \%)$ & 0.812 \\
NSTEMI, n (\%) & $5(25.0 \%)$ & $9(27.3 \%)$ & 0.748 \\
\hline
\end{tabular}

\section{Details of RA and Stent Placement}

Detailed information on RA techniques and stents placement data was shown in Table 2. The most common RA target vessel was the left anterior descending branch (LAD). There was no left circumflex branch RA in our data. The most commonly employed burr size was $1.5 \mathrm{~mm}(70.0 \%)$, mean burr size was $1.48 \pm 0.14 \mathrm{~mm}$. The mean rotation speed was $152,300 \pm 4,200 \mathrm{rpm}$. The mean number of rotations per patient was $5.3 \pm 2.1$ times and the mean ablation time per RA was $11.3 \pm 3.2$ seconds. The mean ACT time was $300.3 \pm 35.7$ seconds. There was one patient with no reflow after RA.

Table2. Details of RA and stent 


\begin{tabular}{|c|c|c|c|}
\hline & $\begin{array}{l}\text { RA group } \\
(\mathrm{n}=20)\end{array}$ & $\begin{array}{l}\text { Control group } \\
(\mathrm{n}=33)\end{array}$ & $P$ value \\
\hline Target Vessel & & & 0.081 \\
\hline LAD, n (\%) & $17(85.0 \%)$ & $21(63.6 \%)$ & \\
\hline LCX, n (\%) & 0 & $4(12.1 \%)$ & \\
\hline RCA, n (\%) & $3(15.0 \%)$ & $8(24.2 \%)$ & \\
\hline \multicolumn{4}{|l|}{ Burr size $(\mathrm{mm})$} \\
\hline $1.25 \mathrm{~mm}, \mathrm{n}(\%)$ & $4(20.0 \%)$ & 0 & \\
\hline $1.5 \mathrm{~mm}, \mathrm{n}(\%)$ & $14(70.0 \%)$ & 0 & \\
\hline $1.75 \mathrm{~mm}, \mathrm{n}(\%)$ & $2(10.0 \%)$ & 0 & \\
\hline Rotation speed (rpm) & $15.2 \pm 0.4$ & 0 & \\
\hline Rotation number (n) & $5.3 \pm 2.1$ & 0 & \\
\hline Ablation time per RA(sec) & $11.3 \pm 3.2$ & 0 & \\
\hline ACT time (sec) & $300.3 \pm 35.7$ & $295.4 \pm 31.5$ & 0.928 \\
\hline Slow flow/no reflow (n) & $1(5.0 \%)$ & 0 & 0.213 \\
\hline Stent number (n) & $1.94 \pm 0.68$ & $1.78 \pm 0.53$ & 0.443 \\
\hline Total stent length (mm) & $57.5 \pm 21.6$ & $45.4 \pm 11.9$ & 0.091 \\
\hline
\end{tabular}

\section{OCT Based Lesion Modification before and after RA}

OCT showed that the maximum calcium angle, maximum calcium thickness and calcium length was $322.5 \pm 58.8^{\circ}$ vs. $309.4 \pm 63.6^{\circ}, 1.67 \pm 0.27$ vs. $0.86 \pm 0.19 \mathrm{~mm}$, and $41.7 \pm 13.5$ vs. $40.7 \pm 13.3 \mathrm{~mm}$ before and after RA, respectively. Although the difference was not statistically significant, the maximum calcium thickness was lower than pre-RA. We found that in the control group, there was no thrombogenesis during the procedure, but in the RA group, all the target vessels had only white thrombus on OCT after RA, which could confirm platelet activation indirectly, and red thrombus was not found. Representative OCT images of RA-related white thrombus are shown in Figure 1. RA led to calcium fracture in $80 \%$ lesions and dissection in $15 \%$ lesions. Representative OCT images of calcium crack and dissection are shown in Figure 2.

\section{Impact Factors of RA-induced White Thrombus}

OCT showed the average number of white thrombus per lesion after RA was $7.23 \pm 4.4$, and the average length of every white thrombus was $0.51 \pm 0.33 \mathrm{~mm}$. Pearson Correlation was calculated between thrombotic load (number of white thrombus) and other variables (burr size, rotation speed, rotation number, ablation time per RA, ACT, maximum calcium angle, maximum calcium thickness, calcium length, and minimal lumen area). The result showed that the thrombotic load was related to burr size 
$(\mathrm{r}=0.575, P=0.040)$ and rotation number $(\mathrm{r}=0.599, P=0.031)$. Bigger burr size and higher number of rotation would result in higher thrombotic load.

Table 3. OCT Based Lesion Modification before and after RA

\begin{tabular}{llll}
\hline & Before RA & After RA & $P$ vaue \\
\hline Maximum calcium angle $\left(^{\circ}\right)$ & $322.5 \pm 58.8$ & $309.4 \pm 63.6$ & 0.919 \\
Maximum calcium thickness $(\mathrm{mm})$ & $1.67 \pm 0.27$ & $0.86 \pm 0.19$ & 0.171 \\
Calcium length $(\mathrm{mm})$ & $41.7 \pm 13.5$ & $40.7 \pm 13.3$ & 0.719 \\
Minimal lumen CSA $\left(\mathrm{mm}^{2}\right)$ & $1.35 \pm 0.35$ & $1.86 \pm 0.29$ & 0.157 \\
White thrombus (n) & 0 & 20 & \\
Calcium crack (n) & 0 & 16 & \\
Dissection $(\mathrm{n})$ & 0 & 3 & \\
\hline
\end{tabular}

\section{Discussion}

We reported for the first time OCT images of RA induced white thrombosis of severely calcified coronary lesions.

RA played an important role in pre-treat of severely calcified lesions before stenting. Complications of RA included coronary dissection (10.5\%), severe coronary spasm (1.6-6.6\%), acute vascular occlusion (3.1\%), slow-flow/no-reflow (1.2-7.6\%) and coronary artery perforation (0-2\%) [14.15]. Among these complications, no-reflow phenomenon remains serious and common. Potential mechanisms of no-reflow during RA included atheromatous debris embolism, platelet activation, microcirculatory vasospasm and so on [16]. A previous study had proved the effect of RA on platelet activation in an in vitro model [8]. To our knowledge, this is the first report about the effect of RA on platelet aggregation in vivo indirectly proved by OCT imaging of white thrombosis. In one article (Kini et al.) which assessed of the mechanistic effects of rotational and orbital atherectomy in severely calcified coronary lesions by OCT (RA details: burr size $1.68 \pm 0.11 \mathrm{~mm}$, rotation speed $150,000 \mathrm{rpm}$ ) [17], the authors found the similar phenomenon, also in all the lesions treated by RA. They defined this phenomenon as small intimal nodules. But according to the OCT image characteristics (different transmissivity and the clear boundary from intima), combined with the results of the in vitro test, we think it is white thrombus. Future histological studies will be necessary to prove this hypothesis.

In our research, only white thrombus was found after RA, which was same to Kini's article[17]. Red thrombus was not found. The reasons may be that red thrombogenesis was usually be seen after the mixed thrombus completely block the lumen. it often composes the tail of the continuous thrombus. During the RA procedure, there was no enough times to generate red thrombus. 
The thrombotic load is influenced by several factors. In our study, we found bigger burr size and higher number of rotation would result in higher thrombotic load. In invitro model study, Reisman et al. tested freshly collected heparinized blood, which was exposed to a $2.0 \mathrm{~mm}$ Rotablator burr rotating at one of three speeds: $180,000,140,000$, or $0 \mathrm{rpm}$. There were significantly more platelet aggregates of $>20$ microm diameter at higher RA speeds, compared with high-speed RA (180,000rpm), low-speed RA $(140,000 \mathrm{rpm})$ can reduce the platelet aggregation [8]. In our study, we did not find the influence of rotation speed on platelet aggregation. The reason may be that in our center, we use low-medium rotation speed $(140,000-160,000 \mathrm{rpm})$ in all the patients in clinical practice, so the relation of rotation speed and thrombotic load can't be found in the Pearson Correlation Analysis.

The mechanism of RA induced platelet aggregation is not entirely clear. It may be caused by heat generated by RA and RA induced cell damage [18]. These data suggest a proper RA strategy (burr size, rotation speed and ablation numbers) should be used to avoid no-reflow during RA. Williams et al. showed that pretreating platelet-rich plasma with abciximab could decrease the RA induced platelet aggregation [9]. Future work is necessary to the potential of therapeutic options, including additional pharmacotherapy agents with RA to mitigate this effect.

This work was an observational study. We generated a hypothesis from the observations. There were several limitations of our research. First, the study sample number was small. Second, the presence of white thrombus does not directly prove platelet aggregation. Future histological studies and randomized controlled study using different RA strategies will be needed to prove this hypothesis.

\section{Conclusions}

White thrombosis during RA is proved by OCT in vivo. Treating calcified lesions with RA may enhance thrombogenesis. The bigger burr size, a higher number of ablation times, maybe together with higher rotation speed, can influence the thrombotic load. These data suggest a proper RA strategy should be used to avoid no-reflow during RA.

\section{List Of Abbreviations}




\begin{tabular}{ll} 
PCI & percutaneous coronary interventions \\
\hline RA & rotational atherectomy \\
\hline OCT & optical coherence tomography \\
\hline ACT & activated clotting time \\
\hline CSA & cross-sectional area \\
\hline MSA & minimum lesion lumen area \\
\hline LAD & left anterior descending branch \\
\hline ACS & acute coronary syndrome
\end{tabular}

\section{Declarations}

Ethics approval and consent to participate

This study was approved by the ethics at Peking University People's Hospital, all patients provided written informed consent before participation.

\section{Consent for publication}

Not applicable

\section{Availability of data and materials}

The datasets used during the current study are available from the corresponding author on reasonable request.

\section{Competing interests}

The authors declare that they have no competing interests

\section{Funding}

This study was supported by The National Key Research and Development Program of China (No. 2016YFC1301105) and The National Natural Science Foundation of China (No. 81800316). These funds designed the study, collection, and analysis of the data.

\section{Authors' contributions}

Cheng-fu CAO was a major contributor in writing the manuscript. Yu-liang MA and Qi LI analyzed and interpreted the patient data, Jian LIU, Hong ZHAO, and Ming-yu LU collected the patient data, Wei-min WANG was the corresponding author. All authors read and approved the final manuscript 
Acknowledgements

Not applicable

\section{References}

1. Moussa I, Ellis SG, Jones M, et al. Impact of coronary culprit lesion calcium in patients undergoing paclitaxel-eluting stent implantation (a TAXUS-IV sub study). Am J Cardiol 2005; 96: 1242-1247.

2. Go AS, Mozaffarian D, Roger VL, et al. Executive summary: Heart disease and stroke statistics-2013 update: A report from the American heart association. Circulation 2013; 127: 143-152.

3. Généreux P, Madhavan MV, Mintz GS, et al. Ischemic outcomes after coronary intervention of calcified vessels in acute coronary syndromes. Pooled analysis from the HORIZONS-AMI (Harmonizing Outcomes With Revascularization and Stents in Acute Myocardial Infarction) and ACUITY (Acute Catheterization and Urgent Intervention Triage Strategy) TRIALS. J Am Coll Cardiol 2014; 63: 1845-1854.

4. Tran T, Brown M, Lasala J. An evidence-based approach to the use of rotational and directional coronary atherectomy in the era of drug-eluting stents: When does it make sense? Catheter Cardiovasc Interv 2008; 72:650-662.

5. Kuriyama N, Kobayashi Y, Yamaguchi M, et al. Usefulness of rotational atherectomy in preventing polymer damage of everolimus-eluting stent in calcified coronary artery. JACC Cardiovasc Interv 2011; 4:588-589.

6. Hodgson J, Stone G, Lincoff AM, et al. Late stent thrombosis: Considerations and practical advice for the use of drug-eluting stents: A report from the Society for Cardiovascular Angiography and Interventions Drug-Eluting Stent Task Force. Catheter Cardiovasc Interv 2007; 69:327-333.

7. Barbato E, Carri D, Dardas P, et al. European expert consensus on rotational atherectomy. Eurolntervention 2015; 11:30-36.

8. Reisman M, Shuman BJ, Dillard D, et al. Analysis of low-speed rotational atherectomy for the reduction of platelet aggregation. Cathet Cardiovasc Diagn. 1998; 45: 208-214.

9. Williams MS, Coller BS, Väänänen HJ, et al. Activation of platelets in platelet-rich plasma by rotablation is speed-dependent and can be inhibited by abciximab (c7E3 Fab; ReoPro). 1998; 98: 742748.

10. Emanuele B, Didier C, Petros D, et al. European expert consensus on rotational atherectomy. Eurolntervention 2015; 11: 30-36.

11. Tearney GJ, Regar E, Akasaka T, et al. Consensus standards for acquisition, measurement, and reporting of intravascular optical coherence tomography studies: A report from the international working group for intravascular optical coherence tomography standardization and validation. J Am Coll Cardiol 2012; 59:1058-1072.

12. Fujino A, Mintz GS, Matsumura M, et al. A new optical coherence tomography-based calcium scoring system to predict stent underexpansion. Eurolntervention. 2018; 13: e2182-e2189. 
13. Guillermo J, Evelyn R, Takashi A, et al. Consensus Standards for Acquisition, Measurement, and Reporting of Intravascular Optical Coherence Tomography Studies: A Report From the International Working Group for Intravascular Optical Coherence Tomography Standardization and Validation. J Am Coll Cardiol. 2012; 59: 58-72.

14. Javier Benezet, Luis Salvador Díaz de la Llera, Jose María Cubero, et al. Drug-eluting stents following rotational atherectomy for heavily calcified coronary lesions: long-term clinical outcomes. J Invasive Cardiol 2011; 23:28-32.

15. Dardas P, Mezilis N, Ninios V, et al. The use of rotational atherectomy and drug-eluting stents in the treatment of heavily calcified coronary lesions. Hellenic J Cardiol 2011; 52:399-406.

16. Tomey MI, Kini AS, Sharma SK. Current Status of Rotational Atherectomy. JACC Cardiovasc Interv 2014; 7: 345-353.

17. Kini AS, Vengrenyuk Y, Pena J, et al. Optical Coherence Tomography Assessment of the Mechanistic Effects of Rotational and Orbital Atherectomy in Severely Calcified Coronary Lesions. Catheter Cardiovasc Interv 2015; 86: 1024-1032.

18. Reisman M, Shuman BJ, Harms V. Analysis of Heat Generation During Rotational Atherectomy Using Different Operational Techniques. Cathet Cardiovasc Diagn. 1998;44:453-455.

\section{Figures}

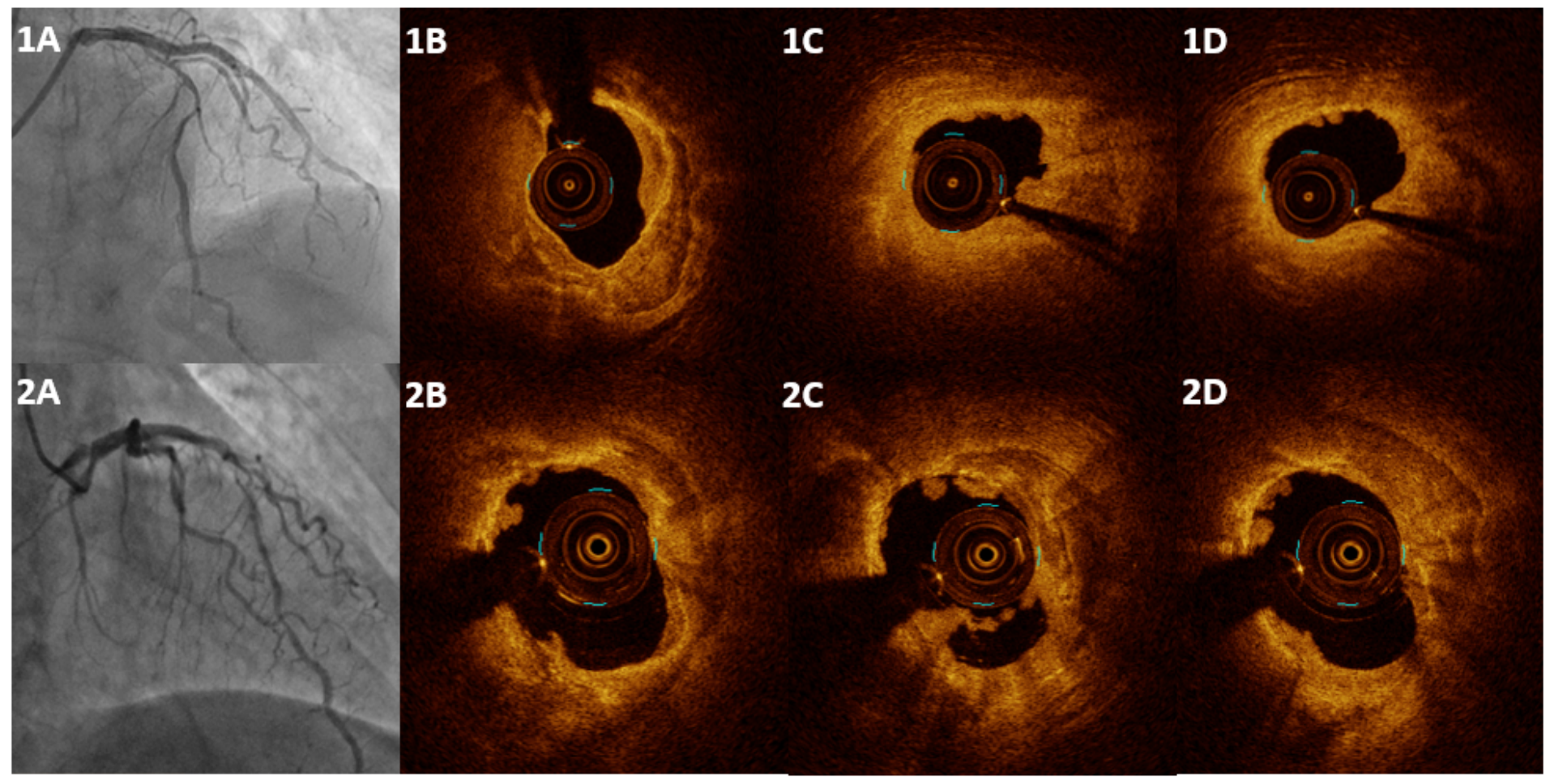

\section{Figure 1}


OCT showed white thrombus after RA in calcified lesions. Case 1: Female, 64Y, coronary artery angiography shows proximal LAD $80 \%$ stenosis with severe calcification (1A). OCT shows maximum calcium angle $360^{\circ}$, maximum calcium thickness $1.34 \mathrm{~mm}$ and minimal lumen area $1.17 \mathrm{~mm} 2$ (1B). RA (1.5mm burr, rotation speed 150,000rpm, ablation time 10sper RA, ablation number 4 times) was received before stent, OCT shows white thrombus after RA (1C, 1D). Case 2: Male, 59Y, coronary artery angiography shows middle LAD 95\% stenosis with severe calcification (2A). OCT catheter and $1.5 \mathrm{~mm}$ balloon did not pass the lesion. So RA (1.5mm burr rotation speed $156000 \mathrm{rpm}$, ablation time 10 sper RA, rotation number 5 times) was received, OCT shows maximum calcium angle $360^{\circ}$, maximum calcium thickness $1.12 \mathrm{~mm}$ and minimal lumen area $1.34 \mathrm{~mm} 2$, many white thrombus after $R A(2 \mathrm{~B}, 2 \mathrm{C}, 2 \mathrm{D}) .1 .75$ burr was further used and implant stents finally.

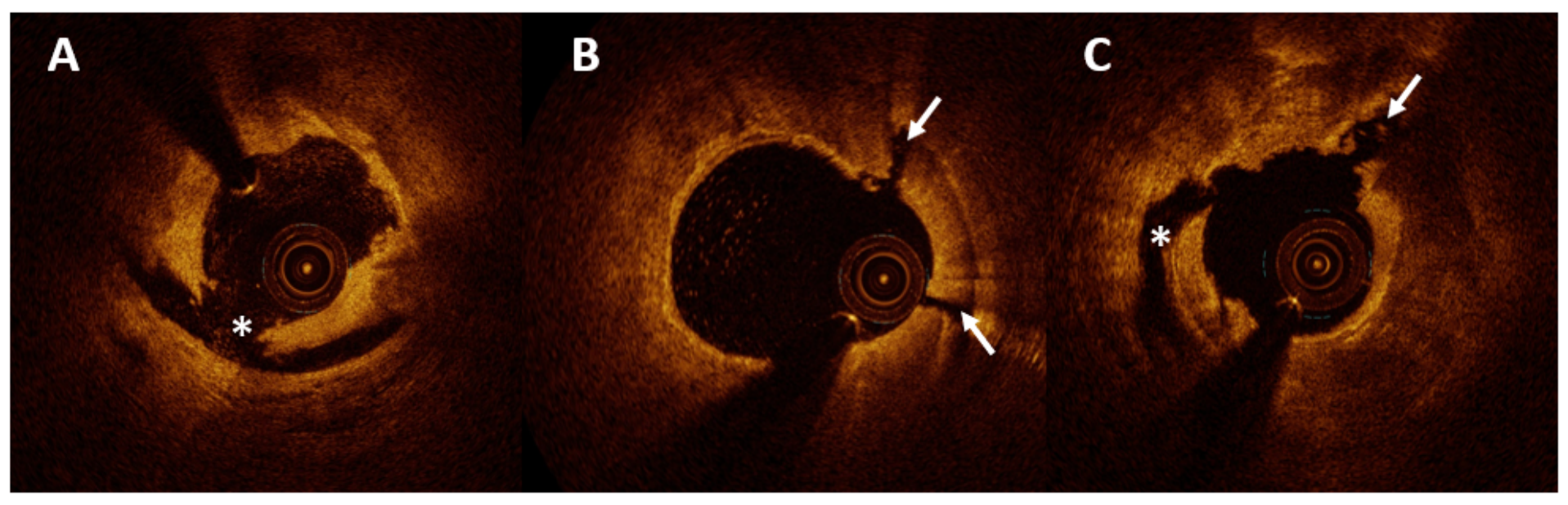

\section{Figure 2}

OCT showed crack and dissection after RA in calcified lesions. OCT showed dissection (asterisks, A and C) and calcification crack (arrows, B and C) 